\title{
A Liver Segmentation Algorithm Based on Wavelets and Machine Learning
}

\author{
Suhuai Luo, Jesse S. Jin, Stephan K. Chalup, Guoyu Qian \\ The University of Newcastle, Australia \\ \{Suhuai.luo,Jesse.jin, Stephan.chalup, Guoyu,qian\}@newcastle.edu.au
}

\begin{abstract}
This paper introduces an automatic liver parenchyma segmentation algorithm that can delineate liver in abdominal CT images. The proposed approach consists of three main steps. Firstly, a texture analysis is applied onto input abdominal CT images to extract pixel level features. Here, two main categories of features, namely Wavelet coefficients and Haralick texture descriptors are investigated. Secondly, support vector machines (SVM) are implemented to classify the data into pixel-wised liver or non-liver. Finally, specially combined morphological operations are designed as a post processor to remove noise and to delineate the liver. Our unique contributions to liver segmentation are twofold: one is that it has been proved through experiments that wavelet features present better classification than Haralick texture descriptors when SVMs are used; the other is that the combination of morphological operations with a pixel-wised SVM classifier can delineate volumetric liver accurately. The algorithm can be used in an advanced computer-aided liver disease diagnosis and surgical planning systems. Examples of applying the algorithm on real CT data are presented with performance validation based on the automatically segmented results and that of manually segmented ones.
\end{abstract}

Keywords- segmentation, machine learning, texture analysis, wavelet, morphology

\section{INTRODUCTION}

An accurate and automatic approach of liver parenchyma segmentation is crucial to a computer-aided liver disease diagnosis and liver surgical planning system such as a system for liver transplantation. However the liver delineation in computer tomography (CT) images is very difficult because of two main reasons. One is that the gray level intensities of liver parenchyma are overlapped with those of the surrounding tissues and organs such as the heart and kidney. The other is that the liver is non-rigid in shape and variant in position. The boundary between the liver and its neighbouring structures is sometimes barely noticeable in CT images [1,2]. Various algorithms have been proposed to deal with the liver segmentation [3-8]. However, great challenges remain in liver segmentation on the aspects of accuracy, robustness, and automation.

In this paper, we present an automatic liver parenchyma segmentation algorithm that can delineate liver in abdominal CT images. In section 2, we describe the algorithm in detail, including its flow diagram and its three major steps. In section 3, we describe the procedure and results of the experiments of applying the proposed algorithm on real CT data as well as the related performance discussion. Finally in section 4 , we conclude by summarising the approach and pointing out possible future pursuits in liver segmentation.

\section{THE ALGORITHM}

In our efforts of developing an automatic segmentation of CT images of the liver, we have focused our attention mainly on three aspects. One is that there are no defined intensity or geometry descriptions to delineate liver in CT images. Therefore, a simple approach such as an intensity threshold will not apply. Another is that both texture features and distribution or resolution features are important in isolating the liver from other surrounding areas. With this consideration, both Wavelet coefficients and Haralick texture descriptors are investigated in feature selection. The last aspect is that the combination of a pixelwised classifier with a shape-wised refiner will deliver a robust yet accurate segmentation of the liver. This leads to our approach of combining SVM classifier with morphological operators. This section details our concepts and implementations in these regards.

\section{A. Algorithm Overview}

The proposed approach of liver segmentation contains three major steps: texture feature extraction, support vector machine classification, and combined morphological operations.

The automatic segmentation process starts with extracting texture features from input CT slices. We propose to use Wavelet coefficients as texture features. In order to compare our approach's performance with that of other approaches, Haralick texture descriptors are also derived to be investigated in the following classification stage. Support vector machines are used to classify each pixel into either liver (with value of 1 assigned) or nonliver (with value of 0 assigned). Here the parameters of the SVM have been derived in a pre-training process, of which the details will be given in the following description. Since the SVM is a pixel-wised classifier, i.e., it classifies the CT slices pixel by pixel, and the classification will not be perfect on test data, there will be both false negative error (FNE) inside the liver parenchyma and false positive error (FPE) outside the liver parenchyma. Therefore, a set of specifically designed morphological operations is used to refine the result of SVM classification to get the delineation of the liver. The following three sections detail these steps.

\section{B. Texture Feature Extraction}

Texture extraction is the process of quantifying the texture patterns within a specified neighbourhood of size $\mathrm{N}$ by $\mathrm{N}$ pixels around a pixel of interest. There mainly exist four categories of texture analysis, namely, structural, statistical, model-based, and transform-based approaches [9]. In our study, two sets of texture features, i.e., Wavelet coefficients and Haralick texture descriptors, are used separately to compare their performances in segmenting the liver. This section describes the definitions and calculations of these features.

\section{1) Haralick texture descriptors}

Haralick texture descriptors [10] are used in statistical texture analysis. The approach represents texture indirectly by the non-deterministic properties that govern the distribution and relationship between the intensities of an image. The approach is based on second-order statistics 
that is the statistics of pairs of pixels. Such statistics is represented with a second-order histogram that is defined as the co-occurrence matrix $\mathrm{P}_{\mathrm{ij}}(\mathrm{d}, \theta)$.

The general idea of a co-occurrence matrix is to represent an image's texture by counting pixel intensity pairs, using a matrix that keeps track of all the pixel-pair counts [11]. $\mathrm{P}_{\mathrm{ij}}(\mathrm{d}, \theta)$, counts all the pixel-pairs within the $\mathrm{N}$ by $\mathrm{N}$ neighbourhood. Here $\mathrm{d}=1, \ldots, \mathrm{N}-1$ is the displacement vector, $\theta=0^{\circ}, 45^{\circ}, 90^{\circ}, 135^{\circ}$ is the angle, i represents the gray-level along the vertical direction and $\mathrm{j}$ represents the gray-level along the horizontal direction.

Once the co-occurrence matrix is calculated for each pixel in the image, nine Haralick texture descriptors can be defined and calculated accordingly as below.

Entropy: measures the randomness of gray-level distribution: $-\sum_{i}^{N} \sum_{j}^{N} P_{i j} \log P_{l j}$

Energy: measures the occurrence of repeated pairs within an image: $\sum_{i}^{N} \sum_{j}^{N} P_{i j}^{2}$

Contrast: measures the local contrast in an image:

$$
\sum_{i}^{N} \sum_{j}^{N}(i-j)^{2} P_{l j}
$$

Sum Average: measures the average of the gray-level within an image: $\frac{1}{2} \sum_{i}^{N} \sum_{j}^{N}\left(i P_{l j}+j P_{l j}\right)$

Variance: measures the variation of gray-level distribution:

$$
\frac{1}{2} \sum_{i}^{N} \sum_{j}^{N}\left(\left(i-\mu_{r}\right)^{2} P_{l j}+\left(j-\mu_{c}\right)^{2} P_{l j}\right)
$$

Correlation: measures a correlation of pixel pairs on gray-levels: $\sum_{i}^{N} \sum_{j}^{N} \frac{\left(i-\mu_{r}\right)\left(j-\mu_{c}\right) P_{i j}}{\sqrt{\sigma_{r}^{2} \times \sigma_{c}^{2}}}$

Maximum Probability (MP): gives the most predominant pixel pair in an image: $\operatorname{Max}_{i, j}^{N, N} P_{i j}$

Inverse Difference Moment (IDM): measures the smoothness of an image: $\sum_{i}^{N} \sum_{j}^{N} \frac{P_{i j}}{1+|i-j|^{2}}$

Cluster Tendency: measures the grouping of pixels that have similar gray-level values: $\left.\sum_{i}^{N} \sum_{j}^{N}\left(i-\mu_{r}+j-\mu_{c}\right)^{2} P_{i j}\right)$

where $\mu_{r}, \mu_{c}, \sigma_{r}^{2}$, and $\sigma_{c}^{2}$ are the means and variance of row and column in $\mathrm{P}_{\mathrm{ij}}(\mathrm{d}, \theta)$ matrix.

\section{2) Wavelet coefficients}

To deal with both the texture characteristics and spatial structures of the liver and its surrounding tissues, Wavelet transform [12] is used to derive inputs to the SVM classifier. Comparing to other transforms such as Fourier [13] and Gabor [14], Wavelet transform has two advantages in segmentation application. One is that it can represent textures at the most suitable scale by varying the spatial resolution. The other is that wavelets best suite for texture analysis in a specific application can be chosen because of a wide range of choices for the wavelet function.
The wavelet transform replaces the Fourier transform's sinusoidal waves by a family generated by translations and dilations of a window called a wavelet. The wavelet transform is defined by

$$
W f(u, s)=\left\langle f, \psi_{u, s}\right\rangle=\int_{-\infty}^{+\infty} f(t) \frac{1}{\sqrt{s}} \psi^{*}\left(\frac{t-u}{s}\right) d t
$$

where the base atom $\psi$ is a zero average function, centered around zero with a finite energy. The family of vectors is obtained by translations and dilatations of the base atom:

$$
\psi_{u, s}(t)=\frac{1}{\sqrt{s}} \psi\left(\frac{t-u}{s}\right)
$$

In image processing applications, the wavelet transform is usually computed with dyadic wavelet transform which is implemented by filter banks. The filtering is done along both rows and columns with pairs of lowpass filter and highpass filter [12]. A one-scale image wavelet decomposition results four blocks of components: (a) the downsampling of the lowpass filtering along both rows and columns; (b) the downsampling of the lowpass filtering along rows and highpass filtering along columns; (c) the downsampling of the highpass filtering along rows and lowpass filtering along columns; and (d) the downsampling of the highpass filtering along both rows and columns. Such filtering or decomposition can be done further on (a), resulting a higher scale representation of the original image.

\section{Support Vector Machine Classification}

Support vector machines (SVMs) [15] are a set of related supervised learning methods used for classification and regression. Viewing input data as two sets of vectors in an n-dimensional space, an SVM will construct a separating hyperplane in that space, one which maximizes the margin between the two data sets.

Assume the training set is $\left\{\left(\mathrm{x}_{\mathrm{i}}, \mathrm{y}_{\mathrm{i}}\right\}, \mathrm{i}=1,2, \ldots 1\right\}$, where $\mathrm{x}_{\mathrm{i}}$ is the input with $x_{i} \in R^{n}, y_{i}$ is the output with $y_{i} \in R$, here $\mathrm{R}=\{-1,+1\}$, and 1 is the number of input samples. Then an optimal hyperplane in canonical form must satisfy the following constraints:

$\omega \phi(x)+b=0$

Where $\mathrm{b} \in \mathrm{R}, \quad \omega$ is a normal vector, and $\phi(x)$ is an inner product induced feature map that maps the input space into a high dimension linear space.

SVMs convert the task of finding the optimal hyperplane into a task of quadratic programming problem as:

$$
\begin{aligned}
& \min \left(\frac{1}{2}\|\omega\|^{2}+C \sum_{i=1}^{l} \xi_{i}\right), \text { subject to } \\
& \left(\omega x_{i}+b\right) \geq 1-\xi_{i}, y_{i} \in\{-1,1\}
\end{aligned}
$$

Where $\xi_{i} \geq 0$ is slack variable, $C>0$ is penalty.

Applying Lagrange multipliers, the optimal quadratic programming problem with the above linear conditions can be solved as the following dual optimal problem:

$$
\begin{aligned}
& \quad \max \left\{\sum_{i=1}^{l} \alpha_{i}-\frac{1}{2} \sum_{i=1}^{l} \sum_{j=1}^{j} \alpha_{i} \alpha_{j} y_{i} y_{j} K\left(x_{i}, x_{j}\right)\right\} \quad, \quad \text { subject } \\
& 0 \leq \alpha_{i} \leq C \text {, and } \sum_{i=1}^{l} \alpha_{i} y_{i}=0
\end{aligned}
$$


Where $\alpha_{i}$ is support value, the $\mathrm{x}_{\mathrm{i}}$ corresponding to $0 \leq \alpha_{i} \leq C$ is called support vector (SV), and the $\mathrm{x}_{\mathrm{i}}$ corresponding to $0<\alpha_{i}<C$ is called normal support vector (noted as NSV).

$$
b=\frac{1}{N_{N S V}} \sum_{x_{i} \in N S V}\left[y_{i}-\sum_{x_{j} \in S V} \alpha_{j} y_{j} K\left(x_{i}, x_{j}\right)\right]
$$

Where $\mathrm{N}_{\mathrm{NSV}}$ is the number of NSV, $K\left(x_{i}, x_{j}\right)$ is kernel function

The training process will derive $\alpha_{i}, b$, and $K\left(x_{i}, x_{j}\right)$. Then the SVM as a classifier can classify any input data $x$ with the following classify function:

$$
f(x)=\operatorname{sign}\left\{\sum_{i=1}^{l} \alpha_{i} y_{i} K\left(x_{i}, x\right)\right\}
$$

\section{Combined Morphological Operations}

A SVM is a pixel-wised classifier. This results two issues that need to be resolved in liver parenchyma segmentation. One is that the classification is not perfect, resulting misclassified pixels both within the liver and outside the liver. The other is that the shape and spatial information is not considered, making the classification sensitive to the noise produced by the misclassified pixels. Therefore, a set of specifically designed binary morphological operations is used to refine the result of SVM classification to get the delineation of the liver.

The combined morphological operation starts with "dilate" and "erode" on the output of pixel-based SVM classifier. Fig. 1(a) presents an original slice of abdominal greyscale CT image. In the image, liver is at the top-left corner, indicated with the white curve. Fig. 1(b) shows the output of SVM on the image. From the figure, it can be seen that the SVM can classify most of the liver pixels correctly but with some errors outside liver as well. By applying "dilate" and then "erode", the dotted pattern inside the liver is converted into connected region as shown in Fig. 1(c). Morphologic operations are described by the shape and size of the structural element used. When a "dilate" or "erode" operator is used, the size of the structural element has to be chosen carefully: if the structural element is too small, multiple regions will form inside the liver; if the structural element is too large, other organs and tissues will be wrongly combined into liver. We used a square structural element with a diameter of 6 pixels for the operation. This value was determined according to both the anatomical structural knowledge of the abdomen and the CT image resolutions.

The second step of the combined morphological operation is to get rid of non-liver areas but keep the liver. This can be easily done by retaining the largest object inside the abdominal volume composed of multiple scans. Fig. 1 (d) shows the outcome.

Up to this point, it can be seen from Fig. 1(d) that there are still some black holes inside the liver. These are the pixels which are liver yet are misclassified as non-liver. To rectify on these positions, "hole filling" operation is applied. Its result is shown in Fig. 1(e).

The last step is to delete the spurs and smooth the contour along edges. Here the "erode" operator is applied first, then the "dilate" operator. Fig. 1(f) shows the final segmented liver area, where the white curve is the liver contour that was manually segmented by radiologist.
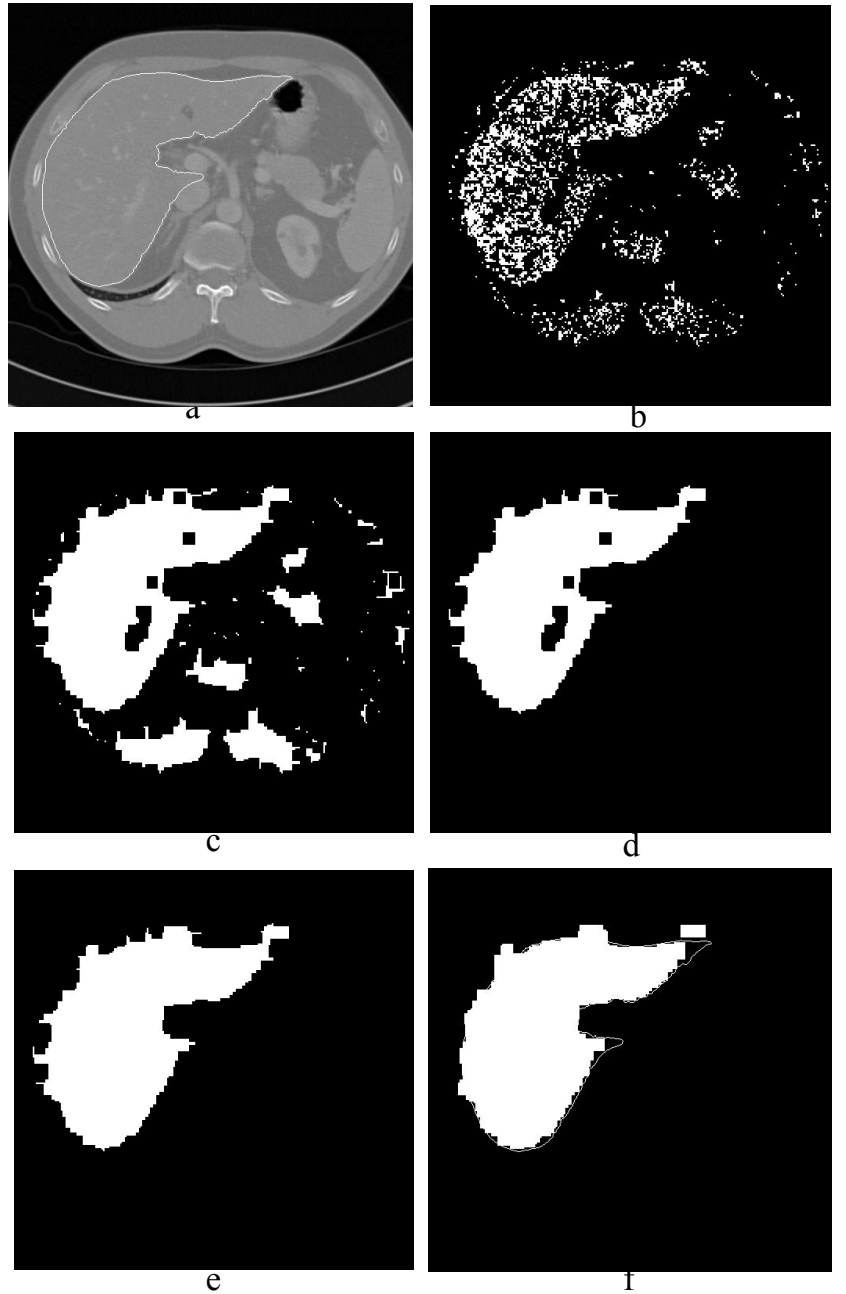

Fig. 1 Refining the output of SVM classification with combined morphological operations

(a) an original slice of abdominal greyscale CT image; (b) output of SVM on the image; (c) output of "dilate" and

"erode"; (d) output of getting rid of non-liver areas; $(e)$ output of "hole filling" operation; $(f)$ output of "smoothing"

operation Note: the white curve in $(a)$ and $(f)$ is the liver boundary manually segmented by radiologist

\section{EXPERIMENTS AND DISCUSSIONS}

The proposed automatic liver parenchyma segmentation algorithm was applied to human abdominal CT images obtained from http://www.sliver07.org/ [16]. All the images were enhanced with contrast agent and scanned in the central venous phase. In designing the support vector machine classifier, an open source software LIBSVM [17] was used as a platform.

Three slices from one data set (Data2) were used as training data. Testing was done on the data set and another data set (Data3). Performance validation was done by comparing the automatically segmented results with the results manually segmented by an experience radiologist. Three metrics are resigned to evaluate the algorithm's performance, of which, the first two are used to evaluate the performance of SVM classifier, and the third is used to evaluate the performance of the proposed automatic liver parenchyma segmentation algorithm as a whole.

False positive volume fraction (FPVF): is defined as the amount of the pixels that are falsely classified by SVM as the liver, as a fraction of the total amount of pixels that 
are manually identified as the liver by radiologist. It can be expressed as: $F P V F=\frac{\left|L_{S V M}-L_{\operatorname{man}}\right|}{\left|L_{\operatorname{man}}\right|}$. Where $\mathrm{L}_{\text {man }}$ denotes the total amount of pixels that are manually identified as liver by radiologist, i.e., the number of 1 's in the classification benchmark. $\mathrm{L}_{\mathrm{SVM}}$ denotes the total amount of the pixels that are classified by SVM as the liver. $\left|L_{S V M}-L_{\text {man }}\right|$ is the set difference between $\mathrm{L}_{\mathrm{SVM}}$ and $\mathrm{L}_{\text {man }}$.

False negative volume fraction (FNVF): is defined as the amount of the pixels that are falsely classified by SVM as the liver, as a fraction of the total amount of pixels that are manually identified as the liver by radiologist. It can be expressed as: $F N V F=\frac{\left|L_{\operatorname{man}}-L_{S V M}\right|}{\left|L_{\operatorname{man}}\right|}$. Where $\left|L_{\text {man }}-L_{S V M}\right|$ is the set difference between $\mathrm{L}_{\operatorname{man}}$ and $\mathrm{L}_{\mathrm{SVM}}$.

True positive volume fraction (TPVF): is defined as the amount of the pixels that are classified as liver by both the proposed automatic liver parenchyma segmentation algorithm and radiologist, as a fraction of the total amount of pixels that are manually identified as the liver by radiologist. It can be expressed as: $F P V F=\frac{\left|L_{\text {proposea }} \cap L_{\text {man }}\right|}{\left|L_{\text {man }}\right|}$. Where $\mathrm{L}_{\text {proposed }}$ denotes the total amount of the pixels that are classified by the proposed automatic liver parenchyma segmentation algorithm as the liver.

Segmentation performance evaluation was done on different data sets and texture features. Table 1 shows the performance metrics of the liver segmentation algorithm.

Table 1 Performance metrics (\%) of the proposed liver segmentation algorithm

\begin{tabular}{|c|c|c|c|c|c|c|}
\hline & \multicolumn{3}{|c|}{ Haralick feature based } & \multicolumn{3}{c|}{ Wavelet based } \\
\cline { 2 - 7 } & FPVF & FNVF & TPVF & FPVF & FNVF & TPVF \\
\hline Data2 & 14.3 & 6.1 & 94.6 & 11.5 & 5.3 & 96.3 \\
\hline Data3 & 18.9 & 8.0 & 92.4 & 15.5 & 7.8 & 94.1 \\
\hline
\end{tabular}

Note that Data2 contains three training images, Data3 contains the images of another subject. From the table we can observe that: SVM classifier alone is not enough to delineate the liver parenchyma. Misclassification happened at both inside the liver (which is measured by FNVF) and outside the liver (which is measured by FPVF). Comparing to Wavelet based approach, Haralick feature based approach resulted in more false positives. On true positive volume fraction, the approach presented about two percent better performance on partly trained data set (i.e., Data2) than non-trained data set. As a whole, Wavelet based approach performed better than Haralick feature based approach. The proposed approach achieved as high as $94.1 \%$ true positive volume fraction on non-trained data set, and $96.3 \%$ on partly trained data set.

\section{CONCLUSION}

With the review of the recent liver segmentation techniques, we have introduced an automatic liver parenchyma segmentation algorithm to delineate liver in abdominal CT images. It is based on efficient texture feature analysis, support vector machines, and combined morphological operations. The approach has two unique contributions to liver segmentation: one is that it has been proved through experiments that wavelet features present better classification than Haralick texture descriptors when SVM is used; the other is that the combination of morphological operations with pixel-wised SVM classifier can delineate volumetric liver accurately. Experiment results on liver segmentation on real CT images have demonstrated the effectiveness of the algorithm. The algorithm can be used in an advanced computer-aided liver disease diagnosis and liver surgical planning system. Future work and further improvements needed for the method include: theoretical and implemental study on the support vector machine classifier to make the pixel based classifier more robust on test data; calculation speed improvement to SVM on both training and testing; and testing on more data.

\section{REFERENCES}

[1] Mortele, K. J., Cantisani, V., Troisi, R., Preoperative Liver Donor Evaluation: Imaging and Pitfalls, Liver Transplantation, Vol 9, No 9, Suppl 1,Sept, 2003: pp S6-S14.

[2] Bogetti, J. D., et al., Accuracy and utility of 3-Dimensional computed tomography in evaluating donors for adult living related liver transplants, Liver Transplantation, Vol. 7, Bo. 8, August, 2001, pp. 687-692.

[3] Bourquain, H., et al., Hepavision2-A Software Assistant for Preoperative Planning in Living Related Liver Transplantation and Oncologic Liver Surgery, Computer Assisted Radiology\&Surgery, June 2002, pp. 341-346.

[4] Meinzer, H.P., Thorn, M., and Cardenas, C., Computerized Planning of Liver Surgery: An Overview, Computers and Graphics, vol. 26, no. 4, 2002, pp. 569-576.

[5] Reitinger, R., Bornik, A., Beichel, R., and Schmalstieg, D., Liver Surgery Planning Using Virtual Reality, IEEE Computer Graphics and Applications, Nov./Dec. 2006, Vol. 26, No. 6, pp. 36-47.

[6] Kainmüller, D., Lange, T., Lamecker, H., Shape constrained automatic segmentation of the liver based on a heuristic intensity model, Proc. MICCAI Workshop 3D Segmentation in the Clinic: A Grand Challenge, T. Heimann, M. Styner, B. van Ginneken (eds.), pp. 109-116, 2007.

[7] Rusko, L., Bekes, G., Nemeth, G., Fidrich, M., Fully automatic liver segmentation for contrast-enhanced CT images, Proc. MICCAI Workshop 3D Segmentation in the Clinic: A Grand Challenge, T. Heimann, M. Styner, B. van Ginneken (eds.), pp. 109-116, 2007.

[8] Liu, J., Hu, Q., Chen, Z., and Heng, P., Adaptive Liver Segmentation from Multi-slice CT Scans, 7th Asian-Pacific Conference on Medical and Biological Engineering(APCMBE) 2008, 22-25 April 2008, Beijing, China, pp. 381-384, 2008.

[9] Materka, A. and Strzelecki, M., Texture analysis methods - a review, Technical Report., Technical University of Lodz, Institute of Electronics, 1998.

[10] Haralick, R. M., Statistical and Structural Approaches to Texture. Proceedings of the IEEE, VOL. 67, NO.5: 786-804, 1979.

[11] Pham, M., Susomboon, R., Disney, T., et al., A Comparison of Texture Models for Automatic Liver Segmentation. SPIE Medical Imaging Conference, San Diego, CA, February 2007, pages 65124E.

[12] Mallat, S., Multifrequency Channel Decomposition of Images and Wavelet Models, IEEE Trans. Acoustic, Speech and Signal Processing, 37, 12, 1989, 2091-2110.

[13] Rosenfeld, A., and Weszka, J., Picture Recognition in Digital Pattern Recognition, K. Fu (Ed.), Springer-Verlag, 135-166, 1980.

[14] Daugman, J., Uncertainty Relation for Resolution in Space, Spatial Frequency and Orientation Optimised by Two-Dimensional Visual Cortical Filters, Journal of the Optical Society of America, 2, 11601169,1985

[15] Cristianini, N., Shawe-Taylor, J., An Introduction to Support Vector Machines and Other Kernel-based Learning Methods, Cambridge University Press, ISBN 0521780195, 2000.

[16] Heimann, T., Styner, M., van Ginneken, B., 3D Segmentation in the Clinic: A Grand Challenge, MICCAI 2007, the 10th Intel Conference on Medical Image Computing and Computer Assisted Intervention, 29 October to 2 November 2007, Brisbane, Australia, pp. 7-15, 2007.

[17] Chih-Chung Chang and Chih-Jen Lin, LIBSVM : a library for support vector machines, 2001, http://www.csie.ntu.edu.tw/ cjlin/libsvm 